EPJ Web of Conferences 95,01009 (2015)

DOI: $10.1051 /$ epjconf/ 20159501009

(C) Owned by the authors, published by EDP Sciences, 2015

\title{
Overview of results from phase I of the Beam Energy Scan pro- gram at RHIC
}

\author{
Daniel McDonald ${ }^{1}$, , on behalf of the STAR Collaboration \\ ${ }^{1}$ University of Houston
}

\begin{abstract}
The first phase of the Beam Energy Scan (BES) program at the Relativistic Heavy Ion Collider (RHIC) was successfully completed during the years 2010, 2011 and 2014, with $\mathrm{Au}+\mathrm{Au}$ collisions at center-of-mass energies $\left(\sqrt{s_{\mathrm{NN}}}\right)$ of 7.7, 11.5, 14.5, 19.6, 27, and $39 \mathrm{GeV}$. The BES has three distinct goals: search for the turning off of the signatures of the Quark Gluon Plasma (QGP), search for the first-order phase transition, and search for the critical point. We report several interesting results that address each of these goals of the BES program.
\end{abstract}

\section{Introduction}

Results from heavy-ion collisions at top energies at RHIC suggest the existence of a phase of nuclear matter known as the Quark Gluon Plasma (QGP), in which quarks and gluons are no longer confined but are asymptotically free [1]. While some progress has been made in understanding the interesting properties of the QGP, little is known about the phase diagram of quark (Quantum Chromodynamics, QCD) matter. The scientific goal of the BES is to elucidate the structures of this phase diagram, significantly increasing the scientific community's knowledge in a very nascent field of study.

Figure 1 shows a conjectured schematic of the QCD phase diagram, with temperature on the y-axis and baryochemical potential, $\mu_{\mathrm{B}}$, on the x-axis. In the upper right is the QGP, and in the lower left is the Hadronic Gas (HG) phase. Posited trajectories at various BES energies are shown in yellow. Theory gives some hints about the structure of the phase diagram. At very low $\mu_{\mathrm{B}}$, lattice QCD calculations predict a smooth crossover transition [2-4]. At higher values of baryochemical potential, QCD-based models predict that there will be a first-order phase transition between the QGP and HG $[5,6]$. At the low- $\mu_{\mathrm{B}}$ end of the first-order phase transition line is a critical point.

The BES has three distinct goals: 1) search for the turning off of the signatures of the QGP, 2) search for the first-order phase transition, and 3) search for the critical point. Table 1 lists details of the data collected during the first phase of the BES. Data were collected at two different interaction points at RHIC: the Solenoid Tracker at RHIC (STAR) and the Pioneering High Energy Nuclear Interaction Experiment (PHENIX).

\footnotetext{
a e-mail: dlmcdona@central.uh.edu
} 


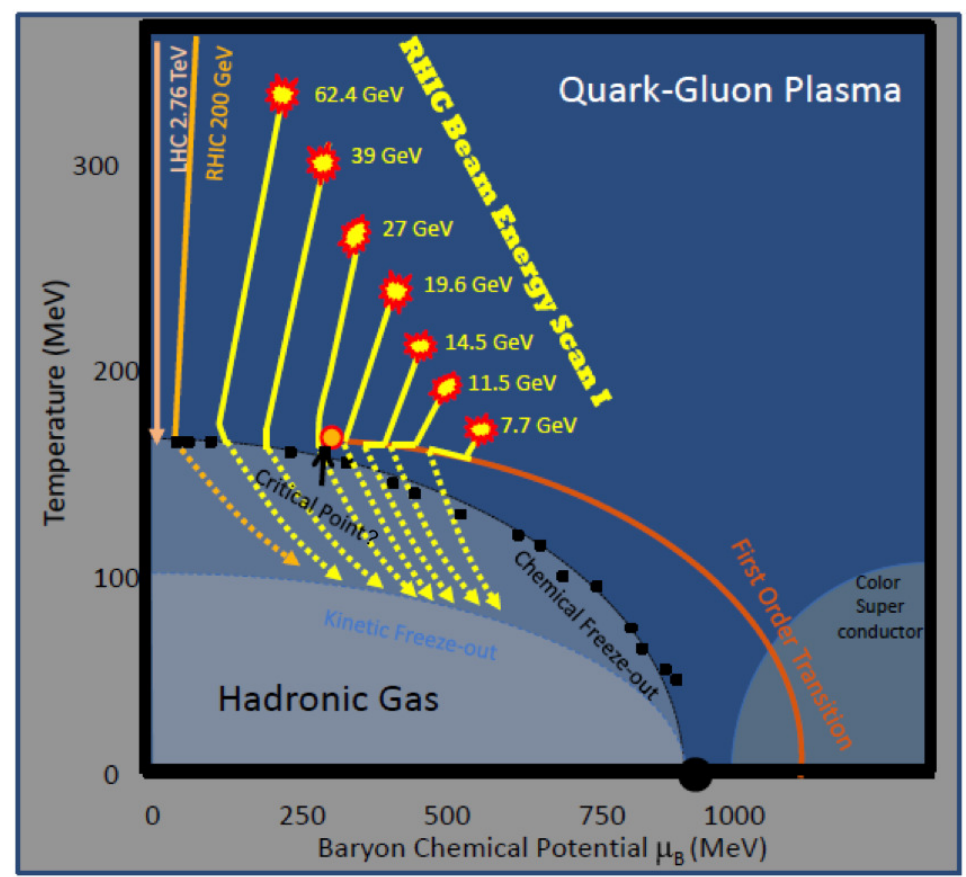

Figure 1. Schematic of the QCD phase diagram with posited trajectories of the RHIC BES program. Yellow trajectories are for illustrative purposes only.

Table 1. Overview of the Beam Energy Scan

\begin{tabular}{lll}
\hline Energy $(\mathrm{GeV})$ & Events $(\mathrm{M})$ & Time (Weeks) \\
\hline 200 & 350 & 11 \\
62.4 & 67 & 1.5 \\
39 & 130 & 2 \\
27 & 70 & 1 \\
19.6 & 36 & 1.5 \\
14.5 & 20 & 3 \\
11.5 & 12 & 2 \\
7.7 & 4 & 4 \\
\hline
\end{tabular}

\section{Search for the turning off of QGP signatures}

\subsection{Nuclear modification factor, $R_{\mathrm{CP}}$}

One of the exciting discoveries at top RHIC energies was the energy loss of high-energy partons and the associated suppression in the production of mesons with high transverse momentum $\left(p_{\mathrm{T}}\right)$ in $\mathrm{Au}+\mathrm{Au}$ collisions [7]. This nuclear modification factor is quantified by $R_{\mathrm{CP}}$, which is the ratio of the number of particles in central A+A collisions to the number of particles in peripheral $\mathrm{A}+\mathrm{A}$ collisions, scaled by the number of binary collisions.

Figure 2 shows $R_{\mathrm{CP}}$ versus $p_{\mathrm{T}}$ at seven different beam energies at STAR. Suppression at high $p_{\mathrm{T}}$ is 


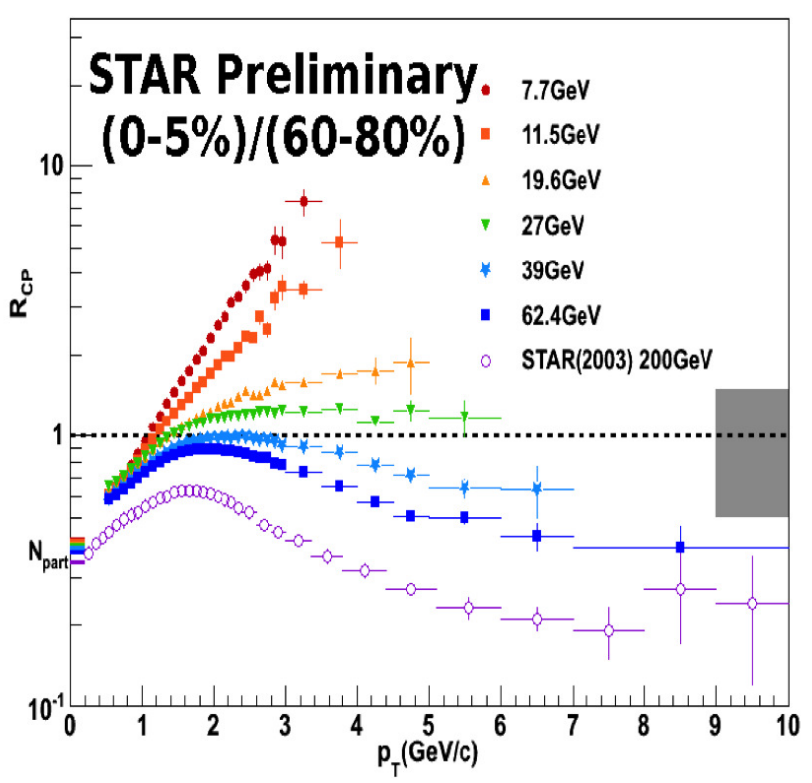

Figure 2. Nuclear modification factor, $R_{\mathrm{CP}}$, as a function of transverse momentum, $p_{\mathrm{T}}$, at different BES beam energies.

indicative of QGP formation. No suppression is seen below $39 \mathrm{GeV}$, but further statistics are needed to extend the $p_{\mathrm{T}}$ reach to lower beam energies.

\subsection{Elliptic flow}

The second-order Fourier coefficient of the azimuthal particle distribution relative to the reaction plane (RP) is indicated by the elliptic flow $v_{2}: v_{2}=\left\langle\cos \left[2\left(\varphi-\Psi_{\mathrm{RP}}\right)\right]\right\rangle$, where $\Psi_{\mathrm{RP}}$ is the azimuth of the reaction plane and $\varphi$ is the azimuthal angle.

Figure 3 shows $v_{2}$ scaled by the number of constituent quarks $\left(\mathrm{n}_{Q}\right)$ as a function of $\mathrm{n}_{Q}$-scaled $\mathrm{m}_{\mathrm{T}}-\mathrm{m}$ for identified hadrons for 0-80\% Au+Au collisions from 7.7-62.4 GeV as published by the STAR collaboration [8]. Scaling by the number of constituent quarks is seen as a possible indicator of partonic behavior. In Fig. 3, $\mathrm{n}_{Q}$ scaling holds for most particles to within $\sim 10 \%$. The $\phi$ meson may not follow the trend of the other identified hadrons at 7.7 and $11.5 \mathrm{GeV}$, but more data are needed before any conclusions can be reached.

Figure 4 shows the difference in $v_{2}$ between particles and their associated antiparticles for $0-80 \%$ $\mathrm{Au}+\mathrm{Au}$ collisions at STAR [9], and includes hybrid [10] and NJL [11] model predictions. For all four particles-protons (circles), $\Lambda$ s (open triangles), kaons (squares), and pions (filled triangles) - the particle and antiparticle $v_{2}$ agree well at $200 \mathrm{GeV}$, and the difference between the particle and antiparticle $v_{2}$ increases as the beam energy decreases. This difference is almost linearly proportional to $\mu_{\mathrm{B}}$, implying a correlation between the $v_{2}$ difference of particles and antiparticles and the net-baryon density.

\subsection{Chiral Magnetic Effect}

The Chiral Magnetic Effect (CME) is a phenomenon where a separation of charges occurs from the large magnetic fields that form in non-central collisions [12]. The CME requires the system to be deconfined; therefore, the turnoff at lower beam energies of the CME would indicate the turning off 

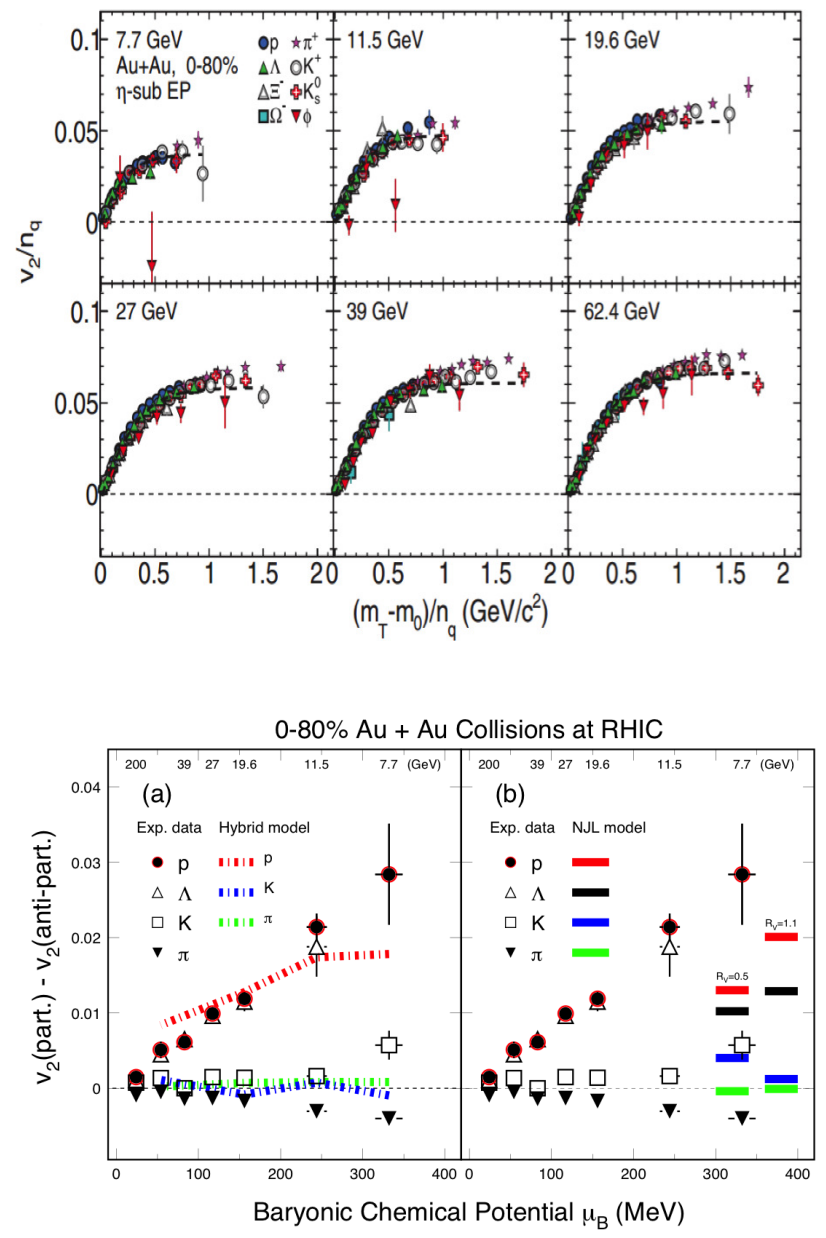

Figure 3. $v_{2}$ scaled by the number of constituent quarks $\left(\mathrm{n}_{Q}\right)$ for identified hadrons versus the $\mathrm{n}_{Q}$-scaled $\mathrm{m}_{\mathrm{T}}-\mathrm{m}$ for 0-80\% Au+Au collisions from 7.7-62.4 $\mathrm{GeV}$ at STAR [8].

Figure 4. The difference in $v_{2}$ between particles and their associated antiparticles for protons (circles), $\Lambda$ s (open triangles), kaons (squares), and pions (filled triangles) versus baryochemical potential and collision energy for $0-80 \% \mathrm{Au}+\mathrm{Au}$ collisions at STAR.

of a QGP signature. Figure 5 shows the three-point correlator, $\gamma$, as a function of centrality in $\mathrm{Au}+\mathrm{Au}$ collisions at STAR from 7.7-62.4 GeV, where opposite charge shown as circles and same charge shown as stars [14]. The value of $\gamma$, a means of experimentally measuring the charge separation, may be sensitive to the CME [15]. One can see that as the energy decreases, the separation diminishes and all but vanishes at $7.7 \mathrm{GeV}$.

\section{Search for the first-order phase transition}

\subsection{Azimuthal anisotropy, $v_{n}$}

A first-order phase transition would be characterized by a region with the lowest compressibility. Hydrodynamic calculations that contain a first-order phase transition indicate that the value of $v_{1}$ of net-baryons can be used as a probe of the phase transition [16]. A non-monotonic variation of the slope of this directed flow would signal a softening of the equation of state.

Figure 6 shows the slope of directed flow $\left(d v_{1} / d y\right)$ near mid-rapidity of antiprotons (upper), protons 


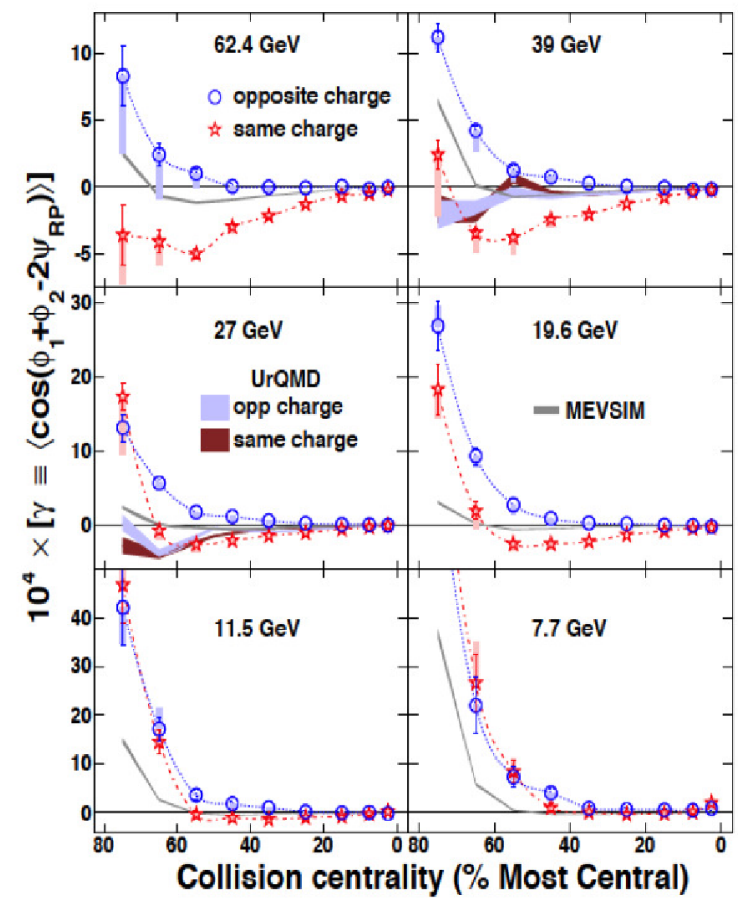

Figure 5. The three-point correlator $(\gamma)$ versus centrality in $\mathrm{Au}+\mathrm{Au}$ collisions from 7.7-62.4 $\mathrm{GeV}$ [14]. Opposite charge is shown as circles and same charge is shown as stars.

(mid), and net protons (lower) for 10-40\% centrality as a function of beam energy in Au+Au collisions at STAR [17]. The proton values change sign between 7.7 and $11.5 \mathrm{GeV}$, have a minimum between 11.5 and $19.6 \mathrm{GeV}$, and increase but remain negative up to $200 \mathrm{GeV}$. The net-proton result also shows a local minimum between 11.5 and $19.6 \mathrm{GeV}$, which could be indicative of the softest region of the equation of state.

When searching for a softening of the equation of state, the $v_{n}$ coefficients are believed to be a promising indicator because they should be proportional to pressure. In addition to the aforementioned $v_{1}$, recent work suggests that $v_{3}$ may also be encouraging. Some model predictions suggest that a hydro phase is not necessary to create $v_{2}$, but that $v_{3}$ is more sensitive to the hydro phase of the fluid created in heavy-ion collisions [18]. Figure 7 shows $v_{3}\{2\}^{2}$ as a function of beam energy for $0-5 \%, 5-10 \%$ and $10-20 \%$ centrality. While multiplicity increases with energy, $v_{3}$ does not increase until $\sim 20 \mathrm{GeV}$, suggesting that this observable warrants further investigation.

\subsection{Average transverse mass, $\mathrm{m}_{\mathrm{T}}$}

The average transverse mass, $\left\langle\mathrm{m}_{\mathrm{T}}\right\rangle$, is related to the temperature of the system. Shown in the left frame of Fig. 8 is $\left\langle\mathrm{m}_{\mathrm{T}}\right\rangle$-m versus beam energy at STAR at midrapidity (stars) for pions (left frame) and kaons (middle frame). Also shown are datapoints from AGS and the LHC (squares) [19]. The right frame of Fig. 8 shows the average transverse energy, $\mathrm{E}_{\mathrm{T}}$, scaled by the charged-particle multiplicity at midrapidity, as prepared by the PHENIX collaboration [20]. $\mathrm{E}_{\mathrm{T}}$ is associated with the energy density. Both $\left\langle\mathrm{m}_{\mathrm{T}}\right\rangle$ and $\mathrm{E}_{\mathrm{T}}$ rise, then flatten out between $\sim 10-100 \mathrm{GeV}$, and then rise again at beam energies $\sim \mathrm{TeV}$. This saturation or plateau of $\left\langle\mathrm{m}_{\mathrm{T}}\right\rangle-\mathrm{m}$ and $\mathrm{E}_{\mathrm{T}}$ could indicate that one is in a mixed-phase regime 

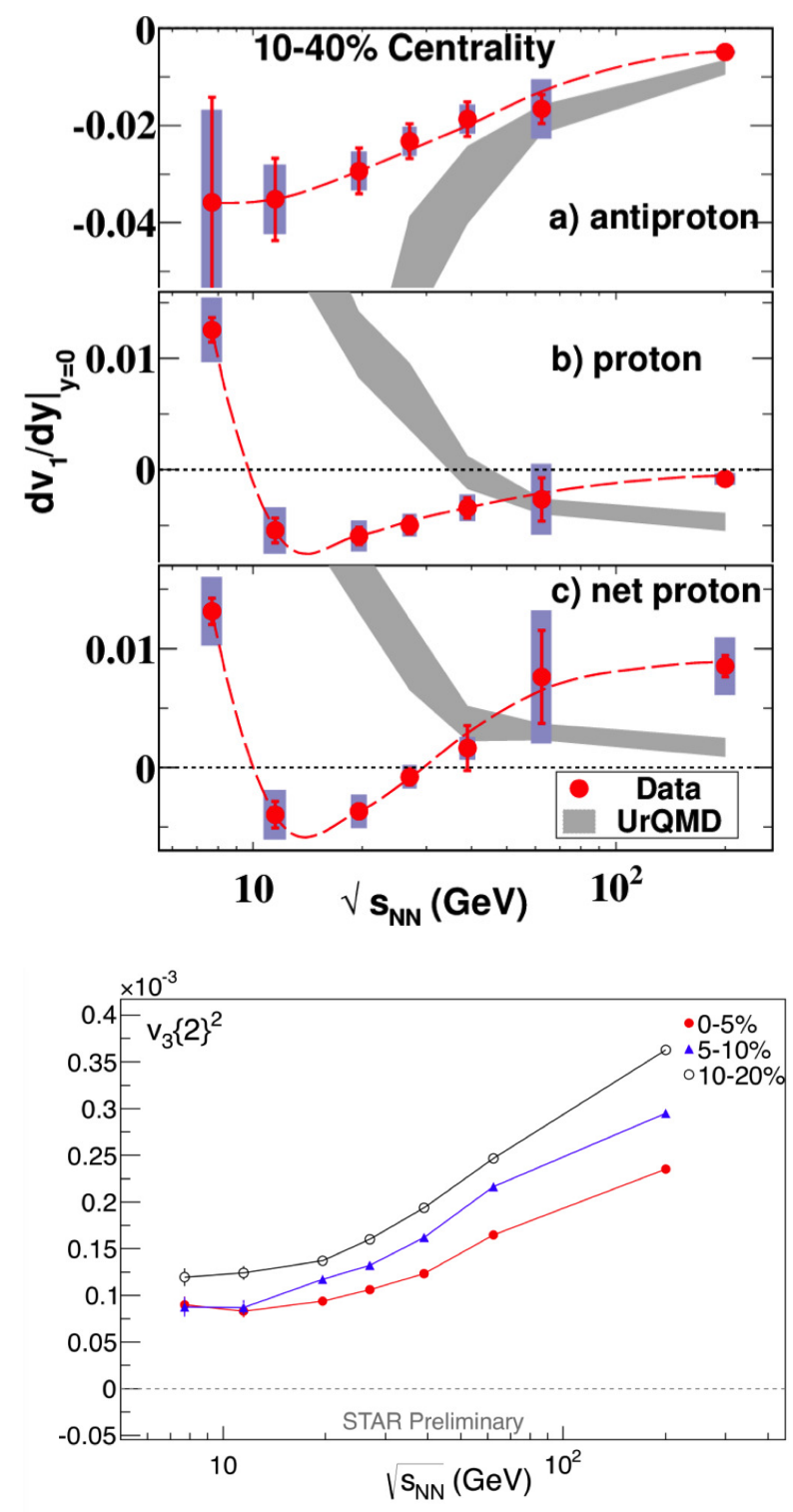

Figure 6. The slope of directed flow $\left(d v_{1} / d y\right)$ near mid-rapidity versus beam energy for mid-central (10-40\%) Au+Au collisions at STAR, as well as UrQMD calculations [17].
Figure 7. Third-order fourier coefficient $v_{3}\{2\}^{2}$ at three different centralities versus beam energy at STAR.

of the QGP and HG, analogous to the plateau in temperature versus entropy in a first-order phase transition [21].

\subsection{Femtoscopy}

The study of two-pion interferometry is typically referred to as HBT (Hanbury Brown and Twiss) studies. HBT has proven to be a precise tool for measuring the pressure anisotropy and lifetime of the system [22]. Since a first-order phase transition would involve a mixed-phase regime where the pressure gradients would be zero, a measurement of the freezeout shape would show non-monotonic behavior near the first-order phase transition [23]. 


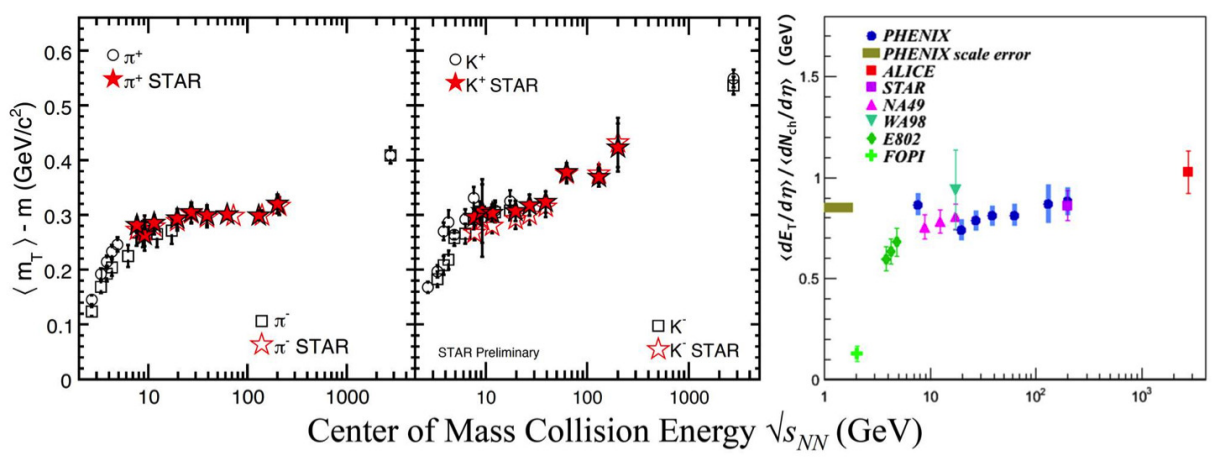

Figure 8. Left panels: The $\left\langle\mathrm{m}_{\mathrm{T}}\right\rangle-\mathrm{m}$ for pions and kaons versus beam energy at RHIC (stars), as well as for $\mathrm{Au}+\mathrm{Au}$ collisions at AGS and $\mathrm{Pb}+\mathrm{Pb}$ collisions at the SPS and LHC [19]. Right panel: The average transverse energy, $\mathrm{E}_{\mathrm{T}}$, scaled by charged particle multiplicity as a function of beam energy from a wide assortment of collaborations [20].

Pion azimuthal HBT allows the study of the coordinate-space almond shape after expansion, given by the kinetic freezeout eccentricity $\epsilon_{\mathrm{F}}$. Early measurements of $\epsilon_{\mathrm{F}}$ hinted at possible non-monotonic behavior [24]. Figure 9 shows $\epsilon_{\mathrm{F}}$ as a function of beam energy, where the STAR datapoints are shown as stars. In addition, data from E895 [25] are shown as squares and data from CERES are shown as a plus sign [26]. Even when changing the rapidity range, STAR does not see any non-monotonic behavior and $\epsilon_{\mathrm{F}}$ follows the general trend of the Boltzmann transport model, UrQMD [27], well.

It has been proposed that one could also search for possible changes in reaction dynamics by looking at different HBT ratios and differences [28]. Figure 10 shows the HBT difference of $\left(R_{\text {out }}\right)^{2}-\left(R_{\text {side }}\right)^{2}$ (left) and the HBT ratio of $\left(R_{\text {side }}-\sqrt{2} \bar{R}\right) / R_{\text {long }}$ for $0-5 \%$ central collisions at PHENIX and STAR (red) and ALICE (blue) as a function of beam energy. These HBT values in Fig. 10 show a peak (left frame) and a minimum (right frame), which could reflect non-monotonic behavior near the first-order phase transition.

\section{Search for the critical point}

\subsection{Higher-order net-particle moments}

Section 3 discusses ongoing research to locate the first-order phase transition of QCD matter. If there is a first-order phase transition, then there should be a critical point where the end of the firstorder phase transition line ends and the transition becomes a crossover [29]. The electric charge, strangeness, and baryon-number susceptibilities, which are directly related to the experimentally measurable cumulants of the multiplicity distributions, should have distinctly different values in the QGP and hadronic phases [30]. In addition, phenomenological models predict that the moments of multiplicity distributions sensitive to the correlation length diverge near the $\mathrm{CP}$, resulting in non-monotonic behavior that should increase in magnitude as the order of the cumulant is increased [31].

Net-proton was used as a proxy of baryon number [32], and net-charge was used as a proxy of electric charge. Figure 11 shows the moment products $\mathrm{S} \sigma$ (upper) and $\kappa \sigma^{2}$ (middle) for net-protons in $0-5 \%$ (circles) and $70-80 \%$ (squares) collisions at STAR [33]. The lower frame shows the values of the moment product $\mathrm{S} \sigma$ scaled by the expectation assuming a Skellam distribution. The Skellam expectations are shown as solid lines. A possible non-monotonic variation of $\kappa \sigma^{2}$ relative to unity 


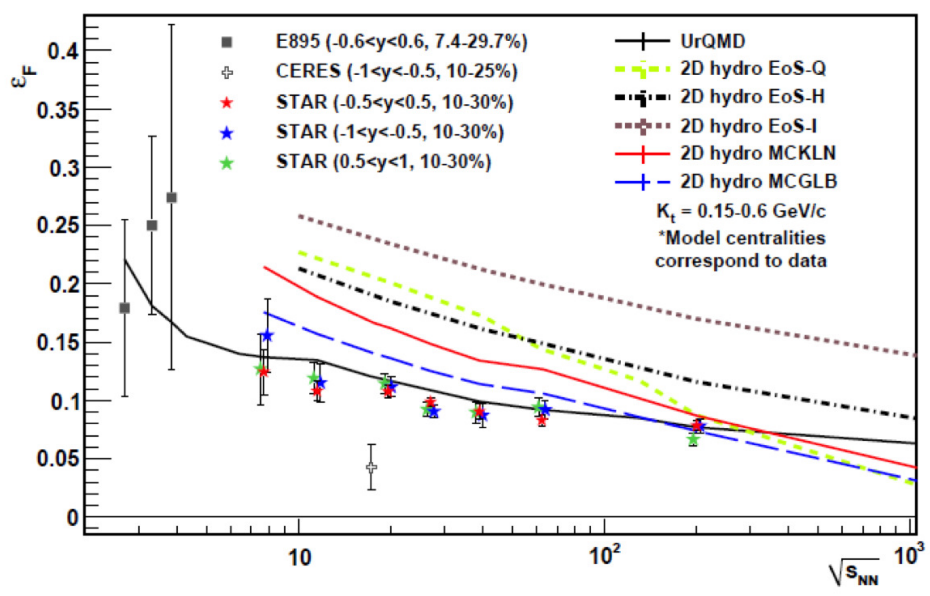

Figure 9. The beam-energy dependence of the kinetic freezeout eccentricity $\epsilon_{\mathrm{F}}$ for $\mathrm{Au}+\mathrm{Au}$ collisions at E895 and STAR and $\mathrm{Pb}+\mathrm{Au}$ collisions at CERES. Three different rapidity ranges (forward, mid, backward) are shown for the STAR energies.

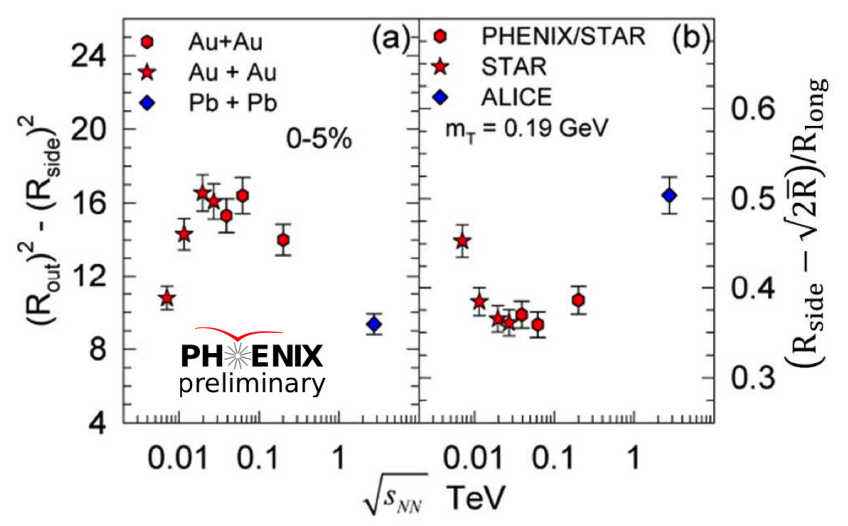

Figure 10. The beam-energy dependence of the HBT difference of $\left(R_{\text {out }}\right)^{2}-\left(R_{\text {side }}\right)^{2}$ (left) and the HBT ratio of $\left(R_{\text {side }}-\sqrt{2} \bar{R}\right) / R_{\text {long }}$ for $0-5 \%$ central collisions at PHENIX and STAR (red) and ALICE (blue).

is not excluded by the existing STAR data. However, the STAR data is in good agreement with the expectation from independent production, which breaks intra-event correlations between the numbers of protons and antiprotons. Higher statistics are necessary to further clarify results.

Figure 12 shows the moment products $\sigma^{2} / \mathrm{M}$ (top), $\mathrm{S} \sigma$ (middle), and $\kappa \sigma^{2}$ (bottom) for net-charge versus beam energy at two different values of centrality at STAR [34]. Solid lines indicate the expectation from a negative binomial distribution (NBD), and dashed lines indicate the expectation assuming Poisson parent distributions. No evidence of non-monotonic behavior of the net-charge moment products is observed within statistical uncertainties.

\section{Dileptons and the chiral transition}

Dileptons are ideal probes of heavy-ion collisions since they are emitted throughout the evolution of the system and are inert to the strong force [35]. In the low invariant mass region (LMR), where 


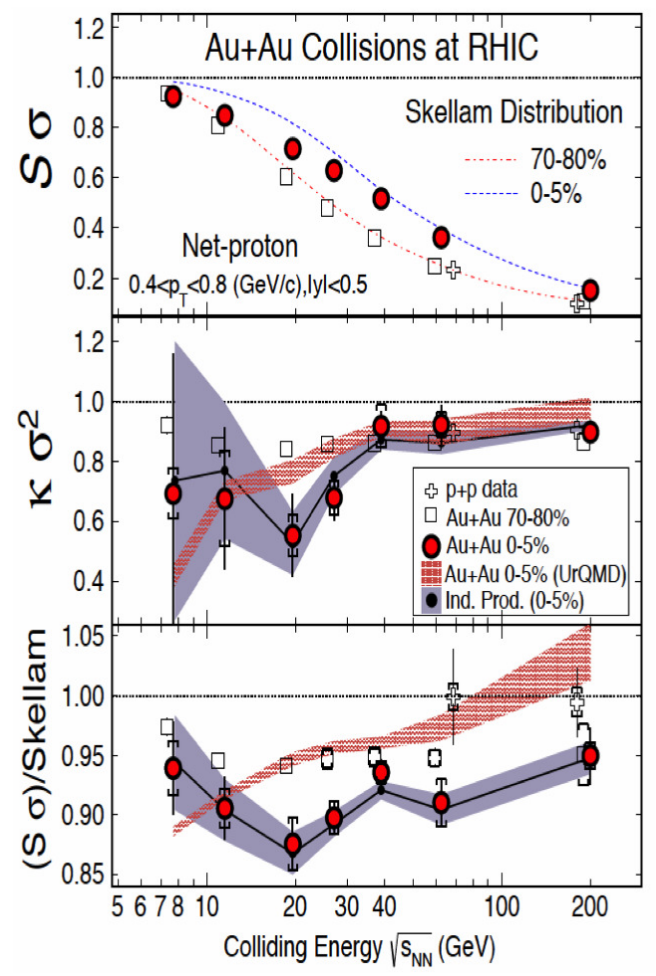

Figure 11. The beam-energy dependence of the net-proton moment products $\mathrm{S} \sigma$ (top) and $\kappa \sigma^{2}$ (middle) at two different values of centrality. The hatched band shows the expectation from UrQMD, and the shaded band shows the expectation assuming independent proton and antiproton production. The bottom frame shows the value of $\mathrm{S} \sigma$ scaled by the Skellam expectation.

$\mathrm{M}_{\mathrm{ee}}<1.1 \mathrm{GeV} / \mathrm{c}^{2}$, the spectral shape of the $\rho$ meson is sensitive to in-medium modifications, making it potentially sensitive to the chiral phase transition [36]. One could also potentially learn about QGP thermal radiation by studying the dilepton measurements in the intermediate invariant mass region (IMR) of $1.1<\mathrm{M}_{\mathrm{ee}}<3.1 \mathrm{GeV} / \mathrm{c}^{2}$ [37].

Figure 13 shows the dielectron invariant mass at four different beam energies at STAR, where the dotted lines are the hadron cocktails without the $\rho$ meson and the shaded bands are the systematic uncertainties. There is no strong beam-energy dependence of the LMR excess. Further comparison with SPS would be possible with energies below $19.6 \mathrm{GeV}$. The excess could spike near the CP because of anomalous increases in the lifetime of the system [38]. Statistics are insufficient for meaningful interpretation of the results in the IMR region.

\section{Summary and Outlook}

We have shown many interesting results from the first phase of the BES at RHIC. When searching for the turning off of QGP signatures, signs of deconfinement remain down to at least $\sim 27 \mathrm{GeV}$. Multiple observables point to potential softening of the equation of state/first-order phase transition in the region of $\sim 20$ to $40 \mathrm{GeV}$, such as $v_{1}, v_{3}$, and the HBT differences and ratios. Further statistics at the lowest BES beam energies are needed before reaching conclusions about the critical point. While there may be some tantalizing hints of interesting phenomena in the RHIC BES, more statistics are needed at the lower beam energies. The associated smaller error bars would allow more definitive conclusions about multiple observables, stressing the importance of a second phase of the BES program at RHIC [19]. 


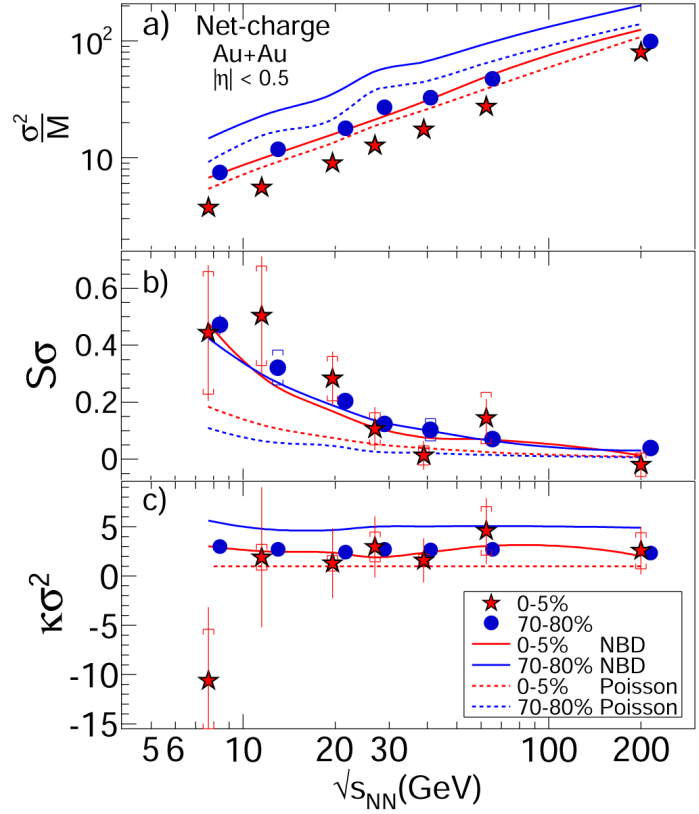

Figure 12. The beam-energy dependence of the net-charge moment products $\sigma^{2} / \mathrm{M}$ (top), $\mathrm{S} \sigma$ (middle), and $\kappa \sigma^{2}$ (bottom) at two different values of centrality. The solid line shows the expectation from a Negative Binomial Distribution (NBD), and the dashed line shows the expectation assuming Poisson parent distributions.

\section{References}

[1] J. Adams et al. [STAR Collaboration], Nucl. Phys. A 757, 28 (2005).

[2] Y. Aoki, S. Borsanyi, S. Durr, Z. Fodor, S. D. Katz, S. Krieg and K. K. Szabo, JHEP 0906, 088 (2009).

[3] S. Borsanyi et al., [Wuppertal-Budapest Collaboration], JHEP 1009, 073 (2010).

[4] A. Bazavov et al., Phys. Rev. D 85, 054503 (2012).

[5] S. Ejiri, Phys. Rev. D 78, 074507 (2008).

[6] E. S. Bowman and J. I. Kapusta, Phys. Rev. C 79, 015202 (2009).

[7] J. Adams et al., [STAR Collaboration], Phys. Rev. Lett. 91, 172302 (2003).

[8] L. Adamczyk et al., [STAR Collaboration], Phys. Rev. C 88, 014902 (2013).

[9] L. Adamczyk et al., [STAR Collaboration], Phys. Rev. Lett. 110, 142301 (2013).

[10] J. Steinheimer, V. Koch and M. Bleicher, Phys. Rev. C 86, 044903 (2012).

[11] J. Xu, T. Song, C. M. Ko and F. Li, Phys. Rev. Lett. 112, 012301 (2014).

[12] D. Kharzeev, Phys. Lett. B 633, 260 (2006).

[13] B. Abelev et al., [ALICE Collaboration], Phys. Rev. Lett. 110, 012301 (2013).

[14] L. Adamczyk et al., [STAR Collaboration], Phys. Rev. Lett. 113, 052302 (2014).

[15] S. Voloshin, Phys. Rev. C 70, 057901 (2004).

[16] H. Stoecker, Nucl. Phys. A 750, 121 (2005).

[17] L. Adamczyk et al., [STAR Collaboration], Phys. Rev. Lett. 112, 162301 (2014).

[18] J. Auvinen, H. Peterson, Phys. Rev. C 88, 064908 (2013).

[19] STAR Collaboration. Studying the Phase Diagram of QCD Mattter at RHIC. SN0598 (2014).

[20] E. O’Brien, [PHENIX Collaboration], Nucl. Phys. A 904, 905 , (2013) 264c.

[21] L. Van Hove, Phys. Lett. B 118, 138 (1982). 


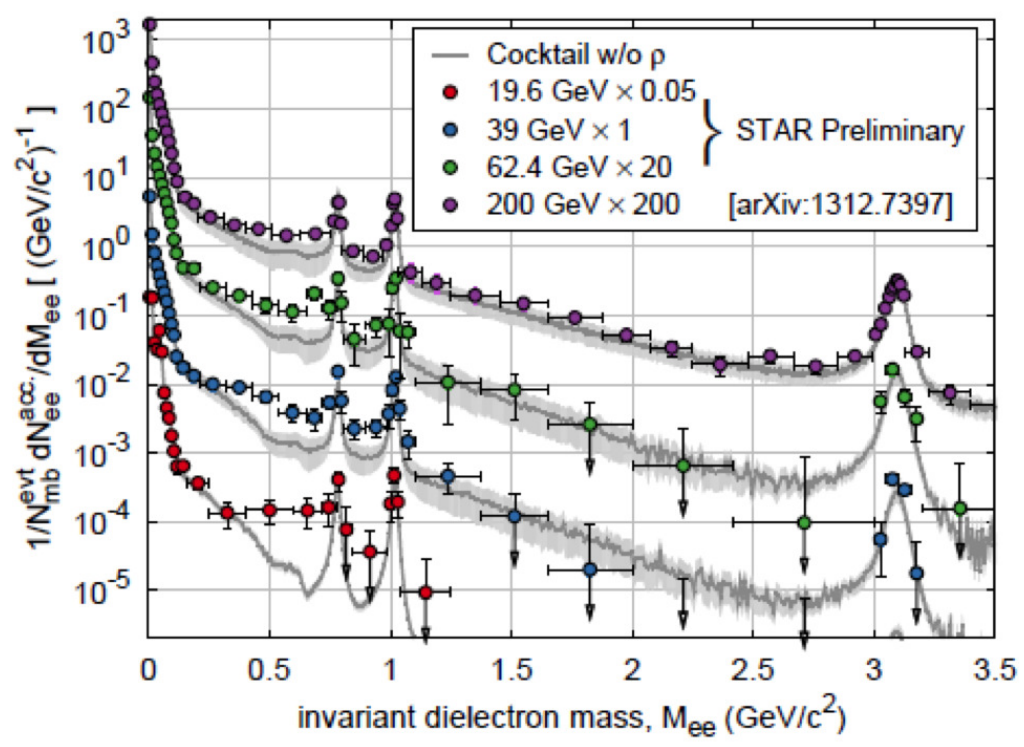

Figure 13. The dielectron invariant mass, $\mathrm{M}_{\mathrm{ee}}$, at four different beam energies. The dotted lines show the hadron cocktails without $\rho$ meson, and the shaded bands are the systematic uncertainties.

[22] L. Adamczyk et al., [STAR Collaboration], (2014), arXiv:1403.4972v1.

[23] P. F. Kolb and U. W. Heinz (2003), arXiv:nucl-th/0305084.

[24] M. A. Lisa, E. Frodermann, G. Graef, M. Mitrovski, E. Mount, H. Petersen, and M. Bleicher,

New J. Phys. 13, 065006 (2011).

[25] M. A. Lisa et al. [E895 Collaboration], Phys. Lett. B 496, 1 (2000).

[26] D. Adamova et al. [CERES Collaboration], Phys. Rev. C 78, 064901 (2008).

[27] H. Petersen, M. Bleicher, S. A. Bass, and H. Stocker (2008), arXiv:0805.0567.

[28] R. Lacey and R. Soltz, Quark Matter 2014.

[29] Y. Aoki, G. Endrodi, Z. Fodor, S. D. Katz and K. K. Szabo, Nature, 443, 675 (2006).

[30] M. Cheng et al., Phys. Rev. D 79, 074505 (2009).

[31] C. Athanasiou et al., Phys. Rev. D 82, 074008 (2010).

[32] Y. Hatta and M. A. Stephanov, Phys. Rev. Lett. 91, 102003 (2003).

[33] L. Adamczyk, et al., [STAR Collaboration], Phys. Rev. Lett. 112, 032302 (2014).

[34] L. Adamczyk, et al., [STAR Collaboration], Phys. Rev. Lett. 113, 092301 (2014).

[35] J. Alam, S. Sarkar, P. Roy, T. Hatsuda and B. Sinha, Annals Phys. 286, 159 (2001).

[36] G. E. Brown and M. Rho, Phys. Rept. 269 (1996) 333.

[37] E. V. Shuryak, Phys. Rept. 61 (1980) 71.

[38] R. Rapp, Adv. High Energy Phys. 2013, 148253 (2013). 